\title{
Nivolumab in Recurrent/Metastatic Squamous Cell Carcinoma of Head and Neck: A Tertiary Cancer Center Experience
}

Ananya Pareek ${ }^{1}$ Apurva A. Patel ${ }^{1}$ Mukesh Kumar ${ }^{1} \quad$ Philip G. Kuttikat $^{1} \quad$ Harshavardhan Annadanam $^{1}$ Shantanu Pendse ${ }^{1}$ Naseer Mohammed ${ }^{1}$ Harsha P. Panchal ${ }^{1}$

${ }^{1}$ Department of Medical Oncology, The Gujarat Cancer and Research Institute, Ahmedabad, Gujarat, India

\section{South Asian J Cancer 2022;11(1):58-61.}

Address for correspondence Apurva A. Patel, MD, DM, Department of Medical Oncology, The Gujarat Cancer and Research Institute, Ahmedabad, Gujarat, India (e-mail: dr.apurvapatel20@gmail.com).

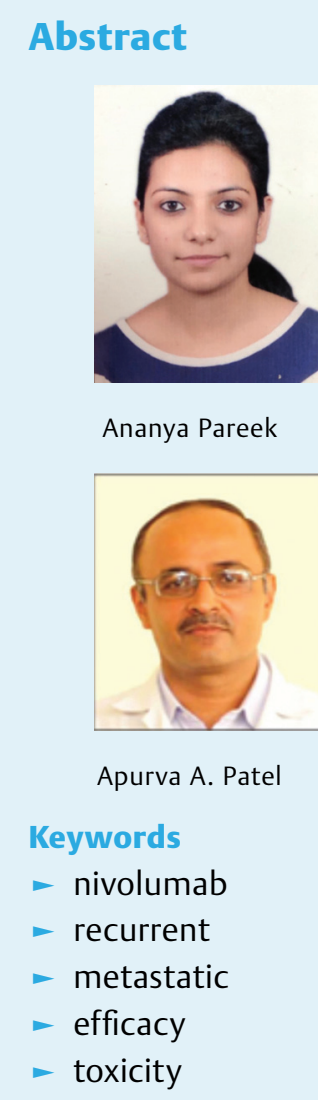

Background Immunotherapy is a proven therapeutic option in recurrent/metastatic head and neck squamous cell carcinoma (R/M HNSCC) after platinum therapy. At present, there are no published Indian data regarding administration of nivolumab in this setting.

Aim The aim of this study is to retrospectively evaluate the efficacy and toxicity of nivolumab in R/M HNSCC among Indian patients who progressed after one or more lines of chemotherapy, including platinum agents.

Methods All patients of R/M HNSCC who received nivolumab between 2/6/2018 to $31 / 3 / 2020$ were assessed retrospectively for the efficacy and toxicity of nivolumab therapy.

Statistical Analysis All the data analysis was performed using IBM SPSS Statistics for Windows, version 25 (IBM Corp., Armonk, N.Y., USA). Descriptive analysis was performed to obtain baseline characteristic of the study sample. Survival analysis was done using the Kaplan-Meier method.

Results Nivolumab therapy was tolerated well, with no new safety concerns, except one (8.3\%) patient experienced grade $3 / 4$ toxicity (gastrointestinal). The clinical benefit rate (CBR) was found to be $66.7 \%$. The median progression-free survival (PFS) was 3 months (95\% Cl; 2.093-3.907), and median overall survival (OS) was 8 months (95\% $\mathrm{Cl}$; 3.731-12.269) from the date of first dose of nivolumab.

Conclusions In our study, efficacy and toxicity were comparable with international data with no new safety concerns. Nivolumab emerged as an astonishing treatment option with tolerable toxicity profile in patients with R/M HNSCC postplatinum therapy, although limited treatment options are available at present.
DOI https://doi.org/10.1055/s-0041-1733317 ISSN 2278-330X

How to cite this article: Pareek A, Patel AA, Kumar M et al. Nivolumab in Recurrent/Metastatic Squamous Cell Carcinoma of Head and Neck: A Tertiary Cancer Center Experience South Asian J Cancer 2022;11(1):58-61. (c) 2021. MedIntel Services Pvt Ltd. All rights reserved.

This is an open access article published by Thieme under the terms of the Creative Commons Attribution-NonDerivative-NonCommercial-License, permitting copying and reproduction so long as the original work is given appropriate credit. Contents may not be used for commercial purposes, or adapted, remixed transformed or built upon. (https://creativecommons.org/licenses/by-nc-nd/ 4.0/)

Thieme Medical and Scientific Publishers Pvt. Ltd., A-12, 2nd Floor, Sector 2, Noida-201301 UP, India 


\section{Introduction}

Squamous cell carcinoma of the head and neck (HNSCC) represents the second most common malignancy in India and is a significant cause of cancer-related morbidity and mortality, accounting for 120,000 new cases diagnosed annually. ${ }^{1}$ Majority of head and neck cancer includes malignancy of oral cavity, pharynx, and larynx. ${ }^{2}$ Platinum refractory recurrent or metastatic head and neck squamous cell carcinoma (R/M HNSCC) patients have poor extended outcomes along with restricted treatment options such as cetuximab. ${ }^{3}$ The median survival of patients whose disease has progressed within 6 months of platinum-based chemotherapy is usually 1 year or less. ${ }^{4}$ The upregulation of programmed death ligands (PD-L1 and PD-L2) results in loss of function of cytotoxic T-lymphocytes, leading to $\mathrm{R} / \mathrm{M}$ HNSCC. ${ }^{5}$ Nivolumab is a fully human Ig4 anti PD-1 monoclonal antibody that binds to programmed death (PD)-1 receptor, which is expressed on T cells, inhibiting the PD-L1 and PD-L2 ligands and PD-1 receptor interaction. ${ }^{6}$ Checkmate 141 has led to its establishment as a further treatment option in patients with platinum refractory advanced disease. ${ }^{7}$

The contemporary availability, high cost, and occurrence of immune-related adverse events are the main factors leading to less universal adaption of immunotherapy in India. ${ }^{8}$ At present, there is no published Indian data regarding administration of nivolumab in R/M HNSCC. Thus, we designed a retrospective analysis of patients who received immunotherapy for the same at our center over the past 2 years.

\section{Material and Methods}

\section{Patient Selection}

Patients who had received platinum therapy, histologically confirmed, and R/M HNSCC (oral cavity, pharynx, or larynx) not pertinent to curative treatment.

\section{Methods}

Records of all patients with R/M HNSCC who have received anti PD-1 antibody nivolumab $(3 \mathrm{mg} / \mathrm{kg}$ ) were extracted retrospectively using hospital management software and reviewed thoroughly. Missing information was also retrieved from medical case files and telephonic conversation with relatives. Patients who received $<8$ weeks or four cycles of nivolumab were excluded from the study.

\section{Treatment}

Nivolumab was given at a dose of $3 \mathrm{mg} / \mathrm{kg}$ once every 2 weeks by intravenous (IV) infusion. Treatment was continued till progression or unacceptable toxicity. Response was assessed clinically after each cycle and radiologically after 2 months or four cycles using immune response criteria in solid tumors (iRECIST).

\section{Statistical Analysis}

All the data analysis was performed using IBM SPSS Statistics for Windows, version 25 (IBM Corp., Armonk, N.Y., USA).
Frequency distribution and cross-tabulation was performed to prepare the tables. Descriptive analysis was performed to obtain baseline characteristic of the study sample. Quantitative data are expressed as mean, and median with standard deviation, whereas categorical data are expressed as number and percentage. Levene's test for equality of variances was performed using independent sample $t$-test for comparison of recurrent/metastatic diseases. Survival analysis was done using the Kaplan-Meier method. $p$-value of $<0.05$ is considered as significant.

\section{Results}

\section{Baseline Characteristics}

Records of 14 patients with R/M HNSCC who received nivolumab were retrieved from $2 / 6 / 2018$ to $31 / 3 / 2020$. Among these patients, two patients received $<4$ cycles; hence, they were excluded from the study. The remaining 12 patients formed the study cohort. The baseline characteristics of these patients are described in - Table 1 . The median age was 57 years, with $25 \%$ of patients aged $>60$ years; minimum and maximum age were 48 and 70 years, respectively. Majority of the patients (91.7\%) were male. Six (50\%) had Eastern Cooperative Oncology Group (ECOG) performance status of 0 and were able to carry out full activities without any restrictions. Oral cavity formed the major primary site in six (50\%) patients, followed by pharynx in five (41.7\%) and larynx in one (8.3\%). Nine (75\%) patients received single line, two (16.7\%) received two lines, and one patient received three lines of systemic chemotherapy previously.

Eight (66.7\%) patients had recurrent, and four (33.3\%) patients had metastatic disease. Platinum refractory disease was present in 11 (91.7\%) patients in cohort.

\section{Treatment Related Toxicity}

Among the study cohort, fatigue was the most commonly experienced toxicity in 10 (83.3\%), followed by hypoalbuminemia in three $(25 \%)$ patients. The adverse events experienced by study cohort have been detailed in - Table 2. Most of the adverse events belonged to grade $1 / 2$, except one ( $8.3 \%$ ) patient experiencing grade $3 / 4$ toxicity (gastrointestinal), for which treatment was stopped. Least common toxicities observed were vomiting and hypothyroidism (-Table 2).

\section{Clinical Benefit and Response Assessment}

A clinical benefit, which is defined as nonprogression of the disease at 3 months of therapy, was present in 8/12 patients converting to a clinical benefit rate (CBR) of $66.7 \%$. At the first response assessment after four cycles of nivolumab, six patients had stable disease (SD) (50\%), disease progression was present in three (25\%) patients, and only one (8.3\%) patient had partial response. During the course of treatment, two patients (16.6\%) had pseudoprogression and none had hyperprogression (-Table 3 ).

\section{Progression-free Survival and Overall Survival}

Progression-free survival (PFS) was defined as the period from the start of nivolumab therapy to the date of radiological/clinical progression of disease or death due to 
Table 1 Characteristics at baseline and previous therapy

\begin{tabular}{|c|c|}
\hline Characteristics & $n(\%)$ \\
\hline Age (years), median (range) & $57(48-70)$ \\
\hline Male gender & $11(91.7)$ \\
\hline \multicolumn{2}{|l|}{ ECOG performance status score } \\
\hline 0 & $6(50)$ \\
\hline 1 & $4(33.3)$ \\
\hline 2 & $2(16.7)$ \\
\hline \multicolumn{2}{|l|}{ Site of primary tumor } \\
\hline Oral cavity & $6(50)$ \\
\hline Pharynx & $5(41.7)$ \\
\hline Larynx & $1(8.3)$ \\
\hline \multicolumn{2}{|c|}{ No. of previous lines of systemic therapy } \\
\hline 1 & $9(75)$ \\
\hline 2 & $2(16.7)$ \\
\hline$\geq 3$ & $1(8.3)$ \\
\hline \multicolumn{2}{|l|}{ Disease status } \\
\hline Recurrent & $8(66.7)$ \\
\hline Metastatic & $4(33.3)$ \\
\hline \multicolumn{2}{|l|}{ Platinum refractory } \\
\hline Yes & $11(91.7)$ \\
\hline No & $8(8.3)$ \\
\hline
\end{tabular}

Table 2 Treatment-related adverse events

\begin{tabular}{|l|l|l|}
\hline Event & Grade $\mathbf{1}$ or $\mathbf{2} \mathbf{n}(\%)$ & Grade $\mathbf{3}$ or $\mathbf{4} \mathbf{n}(\%)$ \\
\hline Fatigue & $10(83.3)$ & 0 \\
\hline Hypoalbuminemia & $3(25)$ & 0 \\
\hline Asthenia & $2(16.7)$ & 0 \\
\hline Anemia & $2(16.7)$ & 0 \\
\hline Diarrhea & $2(16.7)$ & 0 \\
\hline Hypercortisolism & $2(16.7)$ & 0 \\
\hline Colitis & 0 & $1(8.3)$ \\
\hline Vomiting & $1(8.3)$ & 0 \\
\hline Hypothyroidism & $1(8.3)$ & 0 \\
\hline
\end{tabular}

Table 3 Response assessment at the end of treatment (iRECIST)

\begin{tabular}{|l|l|}
\hline Dimension & Response $\mathbf{n}(\%)$ \\
\hline Complete response & 0 \\
\hline Partial response & 0 \\
\hline Stable disease & $2(16.7)$ \\
\hline Unconfirmed pseudoprogression & $1(8.3)$ \\
\hline Progressive disease & $10(83.3)$ \\
\hline Hyperprogression & $1(8.3)$ \\
\hline
\end{tabular}

Abbreviation: iRECIST, immune response criteria in solid tumors.

any cause. The overall survival (OS) in this study cohort was defined as period from the start of nivolumab therapy to the date of death, due to any cause, or the date of last assessment, that is, 31/03/2020. The median PFS of the study cohort was
3 months (95\% CI; 2.093-3.907), and median overall survival (OS) was 8 months $(95 \% \mathrm{Cl} ; 3.731-12.269)$ from the date of first dose of nivolumab.

The outcomes significantly improved among eight patients who achieved clinical benefit. At the time of data cutoff, 10 patients had progressive disease of whom six patients had died and four patients are receiving subsequent lines of chemotherapy. Two patients out of 12 had SD and are continued on nivolumab therapy.

\section{Discussion}

Evasion of immune system is considered as one of the hallmark features of cancer, which permits survival of cancer cells and progression of disease. ${ }^{9}$ Lymphocytes infiltrating the tumor microenvironment in HNSCC has been shown to have impaired function. ${ }^{10}$ The historic discovery of immune checkpoint inhibitors has emerged as a ray of hope for patients with R/M HNSCC, where treatment options are scarce. ${ }^{11,12}$ The main limiting factor in widespread use of immunotherapy is its high cost, especially in a developing country like India. ${ }^{13}$ Indian data regarding the administration of nivolumab in R/M HNSCC is lacking; hence, publication of real-world analysis of the same would help our colleagues to better understand the effectiveness of this drug in our population. Here, we demonstrate our experience of administering nivolumab in 12 patients of $\mathrm{R} / \mathrm{M}$ HNSCC who progressed after platinum therapy.

In our cohort of R/M HNSCC, oral cavity formed the major primary site in $50 \%$ of the patients. Therapy with nivolumab was tolerated well, except for one patient (8.3\%), who developed grade III gastrointestinal toxicity. There were studies that showed incidence of grade III/IV toxicities with immunotherapy to be in range of 7 to $20 \%{ }^{14}$ Immunotherapymediated adverse events differ from those seen in cytotoxic chemotherapies, are infrequent, and resolve rapidly with initiation of treatment. ${ }^{15}$ The most common adverse effect observed in our study group was fatigue (83.3\%). As many patients achieve extended disease stabilization in lieu of response achievement, CBR was chosen as an end point. ${ }^{16}$ In our study, CBR achieved was $66.7 \%$.

The median PFS was 3 months and median OS was 8 months, which is in comparison with checkmate 141 trial. ${ }^{17}$ In random cohorts of other solid tumors that received immunotherapy, the median PFS is in the range of 2 to 4 months and median OS is in the range of 6 to 12 months in recurrent or metastatic setting. ${ }^{18-20}$

Nivolumab was well-tolerated with no new safety concerns and nil mortality. We were not able to find any clinical parameter predicting response to immunotherapy in our study cohort. The main limiting factors of our study are small sample size and retrospective analysis. More prospective studies are required to validate the same.

\section{Conclusion}

There is paucity of data regarding the use of nivolumab and hereby we present first study depicting real-world analysis 
of the same. The efficacy and toxicity profile of our patients were comparable with international data with no new safety concerns. With very few treatment options in therapeutic armamentarium of R/M HNSCC, nivolumab has emerged as a valuable therapeutic option and should be considered in affordable patients.

\section{Funding}

Nil.

\section{Conflicts of Interest}

None declared.

\section{References}

1 Dar MA, Sharma KK. Burden of cancer in India: GLOBOCAN 2018 Estimates Incidence, Mortality, prevalence and future projections of cancer in India. Burden Cancer India 2019;6:505-514

2 Ferlay J, Soerjomataram I, Dikshit R, et al.Cancer incidence and mortality worldwide: sources, methods and major patterns in GLOBOCAN. Int J Cancer 2012

3 Bernier J. Drug Insight: cetuximab in the treatment of recurrent and metastatic squamous cell carcinoma of the head and neck. Nat Clin Pract Oncol 2008;5(12):705-713

4 Saloura V, Cohen EE, Licitra L, et al. An open-label single-arm, phase II trial of zalutumumab, a human monoclonal anti-EGFR antibody, in patients with platinum-refractory squamous cell carcinoma of the head and neck. Cancer Chemother Pharmacol 2014;73(06):1227-1239

5 Ferris RL. Immunology and immunotherapy of head and neck cancer. J Clin Oncol 2015;33(29):3293-3304

6 Fessas P, Lee H, Ikemizu S, Janowitz T. A molecular and preclinical comparison of the PD-1-targeted T-cell checkpoint inhibitors nivolumab and pembrolizumab. Semin Oncol 2017;44(02):136-140

7 Ferris RL, Blumenschein G Jr, Fayette J, et al. Nivolumab vs investigator's choice in recurrent or metastatic squamous cell carcinoma of the head and neck: 2-year long-term survival update of CheckMate 141 with analyses by tumor PD-L1 expression. Oral Oncol 2018;81:45-51

8 Verma V, Sprave T, Haque W, et al. A systematic review of the cost and cost-effectiveness studies of immune checkpoint inhibitors. J Immunother Cancer 2018;6(01):128
9 Ling DC, Bakkenist CJ, Ferris RL, Clump DA. Role of immunotherapy in head and neck cancer. Semin Radiat Oncol 2018;28(01): $12-16$

10 de Ruiter EJ, Ooft ML, Devriese LA, Willems SM. The prognostic role of tumor infiltrating T-lymphocytes in squamous cell carcinoma of the head and neck: A systematic review and metaanalysis. Oncolmmunology 2017;6(11):e1356148

11 Kareemaghay S, Tavassoli M. Clinical immunotherapeutic approaches for the treatment of head and neck cancer. Int J Oral Maxillofac Surg 2019;48(04):419-436

12 Dong M, Meng Z, Kuerban K, et al. Diosgenin promotes antitumor immunity and PD-1 antibody efficacy against melanoma by regulating intestinal microbiota. Cell Death Dis 2018;9(10): 1039

13 Pichler R, Loidl W, Pichler M. High economic burden of immunotherapy underlines the need of predictive biomarkers for the individual therapy algorithm in metastatic bladder cancer. Transl Androl Urol 2018;7(Suppl 6):S738-S740

14 Haanen JBAG, Carbonnel F, Robert C, et al.ESMO Guidelines Committee. Management of toxicities from immunotherapy: ESMO Clinical Practice Guidelines for diagnosis, treatment and follow-up. Ann Oncol 2017;28(Suppl 4):iv119-iv142

15 Brahmer J, Reckamp KL, Baas P, et al. Nivolumab versus docetaxel in advanced squamous-cell non-small-cell lung cancer. N Engl J Med 2015;373(02):123-135

16 Gupta VG, Rangaraju RR, Abbas W, Bajpai P, Khetrapal R. Immune checkpoint inhibitors: Real-world experience from India in advanced solid cancers that have progressed on chemotherapy. South Asian J Cancer 2019;8(01):65-68

17 Ferris RL, Blumenschein G Jr, Fayette J, et al. Nivolumab for Recurrent Squamous-Cell Carcinoma of the Head and Neck. N Engl J Med 2016;375(19):1856-1867

18 Garon EB, Rizvi NA, Hui R, et al. KEYNOTE-001 Investigators. Pembrolizumab for the treatment of non-small-cell lung cancer. $\mathrm{N}$ Engl J Med 2015;372(21):2018-2028

19 El Rassy E, Assi T, Bakouny Z, El Karak F, Pavlidis N, Ghosn M. Comparison of second-line treatments of recurrent and/or metastatic squamous cell carcinoma of the head and neck. Future Oncol 2019;15(08):909-923

20 Sharma P, Retz M, Siefker-Radtke A, et al. Nivolumab in metastatic urothelial carcinoma after platinum therapy (CheckMate 275): a multicentre, single-arm, phase 2 trial. Lancet Oncol 2017;18(03): $312-322$ 